\title{
Some Applications of Clifford Algebra in Geometry
}

\author{
Ying-Qiu $\mathrm{Gu}^{*}$ \\ School of Mathematical Science, Fudan University, Shanghai 200433, China
}

\begin{abstract}
In this paper, we provide some enlightening examples of the application of Clifford algebra in geometry, which show the concise representation, simple calculation and profound insight of this algebra. The definition of Clifford algebra implies geometric concepts such as vector, length, angle, area and volume, and unifies the calculus of scalar, spinor, vector and tensor, so that it is able to naturally describe all variables and calculus in geometry and physics. Clifford algebra unifies and generalizes real number, complex, quaternion and vector algebra, converts complicated relations and operations into intuitive matrix algebra independent of coordinate systems. By localizing the basis or frame of spacetime and introducing differential and connection operators, Clifford algebra also contains Riemann geometry. Clifford algebra provides a unified, standard, elegant and open language and tools for numerous complicated mathematical and physical theories. Clifford algebra calculus is an arithmetic-like operation that can be well understood by everyone. This feature is very useful for teaching purposes, and popularizing Clifford algebra in high schools and universities will greatly improve the efficiency of students to learn fundamental knowledge of mathematics and physics. So Clifford algebra can be expected to complete a new big synthesis of scientific knowledge.
\end{abstract}

Keywords: Clifford algebra, geometric algebra, gamma matrix, multi-inner product, connection operator, Keller connection, Spin group, cross ratio

\section{INTRODUCTION}

Clifford algebra originated from the works of H. Grassmann[1], W. Hamilton[2] and W. K. Clifford (1845-1879)[3]. By introducing associative product of vector bases similar to that of quaternion, Clifford defines his own algebra. Clifford called it geometric algebra, and recognized its special significance as an algebraic representation of geometric concepts as conceived by Grassmann. Unfortunately, his deep insight into the geometric foundations of algebra did not survive due to his early death. Mathematicians abstracted Clifford algebra from its geometric origins, and, for the better part of a century, it languished as a minor subdiscipline of mathematics one more algebra

${ }^{*}$ Electronic address: yqgu@fudan.edu.cn 
among so many others[4].

The events that give the greatest impetus to the development of this algebra should be relativistic quantum mechanics established by Dirac, i.e., the present Dirac equation of spinor[5]. The coefficient matrix $\gamma^{\mu}$ of this equation is a concrete Clifford algebra. Further development of the theory of Clifford algebras is associated with a number of famous mathematicians and physicists: R. Lipschitz, T. Vahlen, E. Cartan, E. Witt, C. Chevalley, M. Riesz and others [6-8]. The first person to realize that Clifford algebra is a unified language in geometry and physics should be David Hestenes. He published "space-time algebra" in 1966 and has been working on the promotion of Clifford algebra in teaching and research[9].

As a unified and universal language of natural science, Clifford algebra has the simplicity and clarity like arithmetic. This language has the following unique features[4]:

1. Clifford algebra summarizes the properties of complex numbers and vectors, thus realizing coordinate-free processing of physical equations.

2. Clifford algebra simplifies grad, div, curl, and all of these as a vector derivative that combines the standard system of four Maxwell equations into one equation.

3. Converting geometric concepts into algebraic operations, Clifford algebra deals with problems in curved space-time and flat one in the same way.

4. Since Clifford algebra is isomorphic to matrix algebra, it is very suitable for pedagogical purposes.

Clifford algebra is developed by many mathematicians, physicists and engineers according to their different requirements and knowledge background, so the prosperity situation is similar to "There are a thousand Hamlets in a thousand people's eyes". In this paper, by introducing typical application of Clifford algebra in geometry, we show some special feature and elegance of the algebra.

\section{APPLICATION OF CLIFFORD ALGEBRA IN DIFFERENTIAL GEOMETRY}

There are several definitions for Clifford algebra[10,11]. Clifford algebra is also called geometric algebra. If the definition is directly related to geometric concepts, it will bring great convenience to the study and research of geometry[12]. 
Definition 1 Assume the element of an $n=p+q$ dimensional space-time $\mathbb{M}^{p, q}$ over $\mathbb{R}$ is given by

$$
d \mathbf{x}=\gamma_{\mu} d x^{\mu}=\gamma^{\mu} d x_{\mu}=\gamma_{a} \delta X^{a}=\gamma^{a} \delta X_{a},
$$

where $\gamma_{a}$ is the local orthogonal frame and $\gamma^{a}$ the coframe. The space-time is endowed with distance $d s=|d \mathbf{x}|$ and oriented volumes $d V_{k}$ calculated by

$$
\begin{aligned}
& d \mathbf{x}^{2}=\frac{1}{2}\left(\gamma_{\mu} \gamma_{\nu}+\gamma_{\nu} \gamma_{\mu}\right) d x^{\mu} d x^{\nu}=g_{\mu \nu} d x^{\mu} d x^{\nu}=\eta_{a b} \delta X^{a} \delta X^{b}, \\
& d V_{k}=d \mathbf{x}_{1} \wedge d \mathbf{x}_{2} \wedge \cdots \wedge d \mathbf{x}_{k}=\gamma_{\mu \nu \cdots \omega} d x_{1}^{\mu} d x_{2}^{\nu} \cdots d x_{k}^{\omega}, \quad(1 \leq k \leq n),
\end{aligned}
$$

in which the Minkowski metric in matrix form reads $\left(\eta_{a b}\right)=\operatorname{diag}\left(I_{p},-I_{q}\right)$, and Grassmann basis $\gamma_{\mu \nu \cdots \omega}=\gamma_{\mu} \wedge \gamma_{\nu} \wedge \cdots \wedge \gamma_{\omega} \in \Lambda^{k} \mathbb{M}^{p, q}$. Then the following number with basis

$$
C=c_{0} I+c_{\mu} \gamma^{\mu}+c_{\mu \nu} \gamma^{\mu \nu}+\cdots+c_{12 \cdots n} \gamma^{12 \cdots n}, \quad\left(\forall c_{k} \in \mathbb{R}\right)
$$

together with multiplication rule of basis given in (2.2) and associativity define the $2^{n}$-dimensional real universal Clifford algebra $C \ell_{p, q}$.

Remark 1 The most important condition in the above definition is the multiplication rule of the basis,

$$
\gamma_{\mu} \gamma_{\nu}+\gamma_{\nu} \gamma_{\mu}=2 g_{\mu \nu}, \quad \gamma_{a} \gamma_{b}+\gamma_{b} \gamma_{a}=2 \eta_{a b}
$$

in which $\gamma_{\mu} \gamma_{\nu}$ is called Clifford product or geometric product. Since Clifford algebra is isomorphic to some matrix algebra, by (2.5) $\gamma_{a}$ is equivalent to some special matrices[12]. In practical calculation, we need not distinguish the vector basis from its representation matrix. The relation between the local frame coefficient $\left(h_{a}^{\mu}, l_{\mu}^{a}\right)$ and metric is given by

$$
\begin{gathered}
\gamma^{\mu}=h_{a}^{\mu} \gamma^{a}, \quad \gamma_{\mu}=l_{\mu}^{a} \gamma_{a}, \quad l_{\mu}^{a} h_{b}^{\mu}=\delta_{b}^{a}, \quad l_{\mu}^{a} h_{a}^{\nu}=\delta_{\mu}^{\nu} . \\
h_{a}^{\mu} h_{b}^{\nu} \eta^{a b}=g^{\mu \nu}, \quad l_{\mu}^{a} l_{\nu}{ }_{\nu} \eta_{a b}=g_{\mu \nu} .
\end{gathered}
$$

Remark 2 Assume $\left\{\gamma_{a} \mid a=1,2 \cdots n\right\}$ to be the basis of the space-time, then their exterior product is defined by

$$
\gamma_{a_{1}} \wedge \gamma_{a_{2}} \cdots \wedge \gamma_{a_{k}} \equiv \frac{1}{k !} \sum_{\forall \sigma} \sigma_{a_{1} a_{2} \cdots a_{k}}^{b_{1} b_{2} \cdots b_{k}} \gamma_{b_{1}} \gamma_{b_{2}} \cdots \gamma_{b_{k}}, \quad(1 \leq k \leq n)
$$

In which $\sigma_{a_{1} a_{2} \cdots a_{k}}^{b_{1} b_{2} \cdots b_{k}}$ is permutation function, if $b_{1} b_{2} \cdots b_{k}$ is the even permutation of $a_{1} a_{2} \cdots a_{k}$, it equals 1 . Otherwise, it equals -1 . The above formula is a summation for all permutations, that is, it is antisymmetrization with respect to all indices. The geometric meaning of the exterior 
product is oriented volume of a high dimensional parallel polyhedron. Exterior algebra is also called Grassmannn algebra, which is associative.

Remark 3 By (2.1) and (2.2) we find that, using Clifford algebra to deal with the problems on a manifold or in the tangent space, the method is the same. Unless especially mentioned, we always use the Greek alphabet to stand for the index in curved space-time, and the Latin alphabet for the index in tangent space. We use Einstein's summation convention.

Remark 4 In number (2.4), each grade- $k$ term is a tensor. For example, $c_{0} I \in \Lambda^{0}$ is a scalar, $c_{\mu} \gamma^{\mu} \in \Lambda^{1}$ is a true vector, $c_{\mu \nu} \gamma^{\mu \nu} \in \Lambda^{2}$ ia an antisymmetric tensor of rank-2, which is also called a bivector, and so on. In practical calculation, coefficient and basis should be written together, because they are one entity, such as (2.1) and (2.4). In this form the variables become coordinatefree. The coefficient is the value of tensor, which is just a number table, but the geometric meaning and transformation law of the tensor is carried by basis.

Definition 2 For vector $\mathbf{x}=\gamma_{\mu} x^{\mu} \in \Lambda^{1}$ and multivector $\mathbf{m}=\gamma_{\theta_{1} \theta_{2} \cdots \theta_{k}} m^{\theta_{1} \theta_{2} \cdots \theta_{k}} \in \Lambda^{k}$, their inner product is defined as

$$
\mathbf{x} \odot \mathbf{m}=\left(\gamma_{\mu} \odot \gamma_{\theta_{1} \theta_{2} \cdots \theta_{k}}\right) x^{\mu} m^{\theta_{1} \theta_{2} \cdots \theta_{k}}, \quad \mathbf{m} \odot \mathbf{x}=\left(\gamma_{\theta_{1} \theta_{2} \cdots \theta_{k}} \odot \gamma_{\mu}\right) x^{\mu} m^{\theta_{1} \theta_{2} \cdots \theta_{k}},
$$

in which

$$
\begin{aligned}
& \gamma^{\mu} \odot \gamma^{\theta_{1} \theta_{2} \cdots \theta_{k}} \equiv g^{\mu \theta_{1}} \gamma^{\theta_{2} \cdots \theta_{k}}-g^{\mu \theta_{2}} \gamma^{\theta_{1} \theta_{3} \cdots \theta_{k}}+\cdots+(-1)^{k+1} g^{\mu \theta_{k}} \gamma^{\theta_{1} \cdots \theta_{k-1}}, \\
& \gamma^{\theta_{1} \theta_{2} \cdots \theta_{k}} \odot \gamma^{\mu} \equiv(-1)^{k+1} g^{\mu \theta_{1}} \gamma^{\theta_{2} \cdots \theta_{k}}+(-1)^{k} g^{\mu \theta_{2}} \gamma^{\theta_{1} \theta_{3} \cdots \theta_{k}}+\cdots+g^{\mu \theta_{k}} \gamma^{\theta_{1} \cdots \theta_{k-1}}
\end{aligned}
$$

Theorem 1 For basis of Clifford algebra, we have the following relations

$$
\begin{aligned}
\gamma^{\mu} \gamma^{\theta_{1} \theta_{2} \cdots \theta_{k}} & =\gamma^{\mu} \odot \gamma^{\theta_{1} \theta_{2} \cdots \theta_{k}}+\gamma^{\mu \theta_{1} \cdots \theta_{k}} \\
\gamma^{\theta_{1} \theta_{2} \cdots \theta_{k}} \gamma^{\mu} & =\gamma^{\theta_{1} \theta_{2} \cdots \theta_{k}} \odot \gamma^{\mu}+\gamma^{\theta_{1} \cdots \theta_{k} \mu} \\
\gamma_{a_{1} a_{2} \cdots a_{n-1}} & =\epsilon_{a_{1} a_{2} \cdots a_{n}} \gamma_{12 \cdots n} \gamma^{a_{n}} \\
\gamma_{a_{1} a_{2} \cdots a_{n-2}} & =\frac{1}{2 !} \epsilon_{a_{1} a_{2} \cdots a_{n}} \gamma_{12 \cdots n} \gamma^{a_{n-1} a_{n}} \\
\gamma_{a_{1} a_{2} \cdots a_{n-k}} & =\frac{1}{k !} \epsilon_{a_{1} a_{2} \cdots a_{n}} \gamma_{12 \cdots n} \gamma^{a_{n-k+1} \cdots a_{n}}
\end{aligned}
$$

Proof. Clearly $\gamma^{\mu} \gamma^{\theta_{1} \theta_{2} \cdots \theta_{k}} \in \Lambda^{k-1} \cup \Lambda^{k+1}$, so we have

$$
\gamma^{\mu} \gamma^{\theta_{1} \theta_{2} \cdots \theta_{k}}=a_{1} g^{\mu \theta_{1}} \gamma^{\theta_{2} \cdots \theta_{k}}+a_{2} g^{\mu \theta_{2}} \gamma^{\theta_{1} \theta_{3} \cdots \theta_{k}}+\cdots+a_{k} g^{\mu \theta_{k}} \gamma^{\theta_{1} \cdots \theta_{k-1}}+A \gamma^{\mu \theta_{1} \cdots \theta_{k}}
$$

Permuting the indices $\theta_{1}$ and $\theta_{2}$, we find $a_{2}=-a_{1}$. Let $\mu=\theta_{1}$, we get $a_{1}=1$. Check the monomial in exterior product, we get $A=1$. Thus we prove (2.12). In like manner we prove (2.13). For 
orthonormal basis $\gamma_{a}$, by $(2.13)$ we have

$$
\gamma_{a_{1} a_{2} \cdots a_{n-1}} \gamma_{a_{n}}=\epsilon_{a_{1} a_{2} \cdots a_{n}} \gamma_{12 \cdots n}
$$

Again by $\gamma_{a_{n}} \gamma^{a_{n}}=1$ (not summation), we prove (2.14). Other equations can be proved by antisymmetrization of indices. The proof is finished.

Likewise, we can define multi-inner product $\mathbf{A} \odot{ }^{k} \mathbf{B}$ between multivectors as

$$
\begin{aligned}
\gamma^{\mu \nu} \odot \gamma^{\alpha \beta} & =g^{\mu \beta} \gamma^{\nu \alpha}-g^{\mu \alpha} \gamma^{\nu \beta}+g^{\nu \alpha} \gamma^{\mu \beta}-g^{\nu \beta} \gamma^{\mu \alpha}, \\
\gamma^{\mu \nu} \odot^{2} \gamma^{\alpha \beta} & =g^{\mu \beta} g^{\nu \alpha}-g^{\mu \alpha} g^{\nu \beta}, \quad \ldots
\end{aligned}
$$

We use $\mathbf{A} \odot{ }^{k} \mathbf{B}$ rather $\mathbf{A}{ }^{k} \mathbf{B}$, because the symbol ' $'$ ' is too small to express exponential power. Then for the case $\gamma^{\mu_{1} \mu_{2} \cdots \mu_{l}} \gamma^{\theta_{1} \theta_{2} \cdots \theta_{k}}$, we have similar results. For example, we have

$$
\gamma^{\mu \nu} \gamma^{\alpha \beta}=\gamma^{\mu \nu} \odot{ }^{2} \gamma^{\alpha \beta}+\gamma^{\mu \nu} \odot \gamma^{\alpha \beta}+\gamma^{\mu \nu \alpha \beta}
$$

In $C \ell_{1,3}$, denote the Pauli matrices by

$$
\begin{gathered}
\sigma^{a} \equiv\left\{\left(\begin{array}{ll}
1 & 0 \\
0 & 1
\end{array}\right),\left(\begin{array}{ll}
0 & 1 \\
1 & 0
\end{array}\right),\left(\begin{array}{cc}
0 & -i \\
i & 0
\end{array}\right),\left(\begin{array}{cc}
1 & 0 \\
0 & -1
\end{array}\right)\right\}, \\
\sigma^{0}=\widetilde{\sigma}^{0}=I, \quad \tilde{\sigma}^{k}=-\sigma^{k}, \quad(k=1,2,3) .
\end{gathered}
$$

We use $k, l, j$ standing for spatial indices. Define Dirac $\gamma$-matrix by

$$
\gamma^{a}=\left(\begin{array}{cc}
0 & \tilde{\sigma}^{a} \\
\sigma^{a} & 0
\end{array}\right), \quad \gamma^{5}=\operatorname{diag}(I,-I) .
$$

$\gamma^{a}$ forms the grade- 1 basis of Clifford algebra $C \ell_{1,3}$. In equivalent sense, the representation (2.24) is unique. By $\gamma$-matrix (2.24), we have the complete bases of $C \ell_{1,3}$ as follows,

$$
I, \quad \gamma^{a}, \quad \gamma^{a b}=\frac{i}{2} \epsilon^{a b c d} \gamma_{c d} \gamma^{5}, \quad \gamma^{a b c}=i \epsilon^{a b c d} \gamma_{d} \gamma^{5}, \quad \gamma^{0123}=-i \gamma^{5} .
$$

Based on the above preliminaries, we can display some enlightening examples of application, which show how geometric algebra works efficiently. For a skew-symmetrical torsion $\mathcal{T}_{\mu \nu \omega} \equiv g_{\mu \beta} \mathcal{T}_{\nu \omega}^{\beta}$ in $\mathbb{M}^{1,3}$, by Clifford calculus, we have

$$
\mathcal{T}=\mathcal{T}_{\mu \nu \omega} \gamma^{\mu \nu \omega}=\mathcal{T}_{a b c} \gamma^{a b c}=\mathcal{T}_{a b c} \epsilon^{a b c d} \gamma_{d}\left(i \gamma^{5}\right) \equiv i \gamma_{d} \gamma^{5} \mathcal{T}^{d}=i \gamma_{\alpha} \gamma^{5} \mathcal{T}^{\alpha}
$$

and then

$$
\mathcal{T}^{\alpha}=h_{d}^{\alpha} \mathcal{T}_{a b c} \epsilon^{a b c d}=\mathcal{T}_{\mu \nu \omega} h^{\mu}{ }_{a} h_{b}^{\nu} h_{c}^{\omega} h_{d}^{\alpha} \epsilon^{a b c d}=\frac{1}{\sqrt{g}} \epsilon^{\mu \nu \omega \alpha} \mathcal{T}_{\mu \nu \omega}
$$


where $g=\left|\operatorname{det}\left(g_{\mu \nu}\right)\right|$. So we get

$$
\mathcal{T}_{\mu \nu \omega}=\sqrt{g} \epsilon_{\mu \nu \omega \alpha} \mathcal{T}^{\alpha}, \quad \mathcal{T}_{\mu \nu \omega} \mathcal{T}^{\omega}=0, \quad \mathcal{T}_{\mu \nu}^{\alpha} \mathcal{T}^{\nu}=0
$$

So the skew-symmetrical torsion is equivalent to a pseudo vector in $\mathbb{M}^{1,3}$. This example shows the advantages to combine variable with basis together.

The following example discusses the absolute differential of tensors. The definition of vector, tensor and spinor in differential geometry involving a number of refined concepts such as vector bundle and dual bundle, which are too complicated for readers in other specialty. Here we inherit the traditional definitions based on the bases $\gamma^{a}$ and $\gamma^{\mu}$. In physics, basis of tensors is defined by direct products of grade-1 bases $\gamma^{\mu}$. For metric we have[13]

$$
\begin{aligned}
\mathbf{g} & =g_{\mu \nu} \gamma^{\mu} \otimes \gamma^{\nu}=g^{\mu \nu} \gamma_{\mu} \otimes \gamma_{\nu}=\delta_{\mu}^{\nu} \gamma^{\mu} \otimes \gamma_{\nu} \\
& =\eta_{a b} \gamma^{a} \otimes \gamma^{b}=\eta^{a b} \gamma_{a} \otimes \gamma_{b}=\delta_{a}^{b} \gamma^{a} \otimes \gamma_{b}
\end{aligned}
$$

For simplicity we denote tensor basis by

$$
\otimes \gamma^{\mu_{1} \mu_{2} \cdots \mu_{n}}=\gamma^{\mu_{1}} \otimes \gamma^{\mu_{2}} \otimes \cdots \gamma^{\mu_{n}}, \quad \otimes \gamma_{\mu_{1}}^{\mu_{2} \mu_{3} \cdots \mu_{n}}=\gamma_{\mu_{1}} \otimes \gamma^{\mu_{2}} \otimes \cdots \gamma^{\mu_{n}}, \cdots
$$

In general, a tensor of rank $n$ is given by

$$
\mathbf{T}=T_{\mu_{1} \mu_{2} \cdots \mu_{n}} \otimes \gamma^{\mu_{1} \mu_{2} \cdots \mu_{n}}=T_{\mu_{2} \cdots \mu_{n}}^{\mu_{1}} \otimes \gamma_{\mu_{1}}^{\mu_{2} \mu_{3} \cdots \mu_{n}}=\cdots
$$

The geometrical information of the tensor such as transformation law and differential connection are all recorded by basis $\gamma^{\mu}$, and all representations of rank $(r, s)$ tensor denote the same one practical entity $\mathbf{T}(\mathbf{x}) . T_{\mu \cdots}^{\nu \cdots}$ is just a quantity table similar to $c_{\mu \nu}$ in (2.4), but the physical and geometrical meanings of the tensor $\mathbf{T}$ are represented by basis $\gamma^{\mu}$. Clifford algebra is a special kind of tensor with exterior product. Its algebraic calculus exactly reflects the intrinsic property of space-time and makes physical calculation simple and clear.

For the absolute differential of vector field $\mathbf{A}=\gamma_{\mu} A^{\mu}$, we have

$$
\begin{aligned}
d \mathbf{A} & \equiv \lim _{\Delta \mathbf{x} \rightarrow 0}[\mathbf{A}(\mathbf{x}+\Delta \mathbf{x})-\mathbf{A}(\mathbf{x})] \\
& =\left(\partial_{\alpha} A^{\mu} \gamma_{\mu}+A^{\mu} \mathfrak{d}_{\alpha} \gamma_{\mu}\right) d x^{\alpha}=\left(\partial_{\alpha} A_{\mu} \gamma^{\mu}+A_{\mu} \mathfrak{d}_{\alpha} \gamma^{\mu}\right) d x^{\alpha}
\end{aligned}
$$

We call $\mathfrak{d}_{\alpha}$ connection operator[13]. According to its geometrical meanings, connection operator should satisfy the following conditions:

$1^{\circ}$ it is a real linear transformation of basis $\gamma^{\mu}$, 
$2^{\circ}$ it satisfies metric consistent condition $d \mathbf{g}=0$.

Thus the differential connection can be generally expressed as

$$
\mathfrak{d}_{\alpha} \gamma^{\mu}=-\left(\Pi_{\alpha \beta}^{\mu}+\mathcal{T}_{\alpha \beta}^{\mu}\right) \gamma^{\beta}, \quad \Pi_{\alpha \beta}^{\mu}=\Pi_{\beta \alpha}^{\mu}, \quad \mathcal{T}_{\alpha \beta}^{\mu}=-\mathcal{T}_{\beta \alpha}^{\mu} .
$$

For metric $\mathbf{g}=g_{\mu \nu} \gamma^{\mu} \otimes \gamma^{\nu}$, by metric consistent condition we have

$$
\begin{aligned}
0 & =d \mathbf{g}=d\left(g_{\mu \nu} \gamma^{\mu} \otimes \gamma^{\nu}\right) \\
& =\left[\left(\partial_{\alpha} g_{\mu \nu}\right) \gamma^{\mu} \otimes \gamma^{\nu}+g_{\mu \nu}\left(\mathfrak{d}_{\alpha} \gamma^{\mu}\right) \otimes \gamma^{\nu}+g_{\mu \nu} \gamma^{\mu} \otimes \mathfrak{d}_{\alpha} \gamma^{\nu}\right] d x^{\alpha} \\
& =\left[\left(\partial_{\alpha} g_{\mu \nu}-g_{\nu \beta} \Pi_{\alpha \mu}^{\beta}-g_{\mu \beta} \Pi_{\alpha \nu}^{\beta}\right) d x^{\alpha}-\left(g_{\nu \beta} \mathcal{T}_{\alpha \mu}^{\beta}+g_{\mu \beta} \mathcal{T}_{\alpha \nu}^{\beta}\right) d x^{\alpha}\right] \gamma^{\mu} \otimes \gamma^{\nu}
\end{aligned}
$$

By (2.34), we have

$$
\left(\partial_{\alpha} g_{\mu \nu}-g_{\nu \beta} \Pi_{\alpha \mu}^{\beta}-g_{\mu \beta} \Pi_{\alpha \nu}^{\beta}\right) d x^{\alpha}-\left(g_{\nu \beta} \mathcal{T}_{\alpha \mu}^{\beta}+g_{\mu \beta} \mathcal{T}_{\alpha \nu}^{\beta}\right) d x^{\alpha}=0
$$

Since $d x^{\alpha} \leftrightarrow \delta X^{a}$ is an arbitrary vector in tangent space, (2.35) is equivalent to

$$
\partial_{\alpha} g_{\mu \nu}-g_{\nu \beta} \Pi_{\alpha \mu}^{\beta}-g_{\mu \beta} \Pi_{\alpha \nu}^{\beta}=g_{\nu \beta} \mathcal{T}_{\alpha \mu}^{\beta}+g_{\mu \beta} \mathcal{T}_{\alpha \nu}^{\beta}
$$

(2.36) is a linear nonhomogeneous algebraic equation of $\left(\Pi_{\alpha \beta}^{\mu}, \mathcal{T}_{\alpha \beta}^{\mu}\right)$.

Solving (2.36), we get the symmetrical particular solution 'Christoffel symbols' as follows,

$$
\Pi_{\mu \nu}^{\alpha}=\frac{1}{2} g^{\alpha \beta}\left(\partial_{\mu} g_{\beta \nu}+\partial_{\nu} g_{\mu \beta}-\partial_{\beta} g_{\mu \nu}\right)+\pi_{\mu \nu}^{\alpha}=\Gamma_{\mu \nu}^{\alpha}+\pi_{\mu \nu}^{\alpha},
$$

in which $\Gamma_{\mu \nu}^{\alpha}$ is called Levi-Civita connection determined by metric, $\pi_{\mu \nu}^{\alpha}=\pi_{\nu \mu}^{\alpha}$ is a symmetrical post-metric part of connection. In this paper, the 'post-metric connection' means the parts of connection cannot be determined by metric, i.e., the components $\pi_{\mu \nu}^{\alpha}$ and $\mathcal{T}_{\mu \nu}^{\alpha}$ different from Levi-Civita connection $\Gamma_{\mu \nu}^{\alpha}$. Denote

$$
\mathcal{T}_{\mu \mid \nu \alpha}=g_{\mu \beta} \mathcal{T}_{\nu \alpha}^{\beta}, \quad \pi_{\mu \mid \nu \alpha}=g_{\mu \beta} \pi_{\nu \alpha}^{\beta}, \quad K_{\mu \nu \alpha}=\pi_{\mu \mid \nu \alpha}+\mathcal{T}_{\mu \mid \nu \alpha}
$$

where $K_{\mu \nu \alpha}$ is called contortion with total $n^{3}$ components. Substituting (2.37) and (2.38) into metric compatible condition $(2.36)$, we get $\frac{1}{2}(n+1) n^{2}$ constraints for $K_{\mu \nu \alpha}$,

$$
K_{\mu \nu \alpha}+K_{\nu \mu \alpha}=0=\left(\pi_{\mu \mid \nu \alpha}+\pi_{\nu \mid \mu \alpha}\right)+\left(\mathcal{T}_{\mu \mid \nu \alpha}+\mathcal{T}_{\nu \mid \mu \alpha}\right)
$$

By (2.39), $K_{\mu \nu \alpha}$ has only $\frac{1}{2}(n-1) n^{2}$ independent components. Noticing torsion $\mathcal{T}_{\mu \mid \nu \alpha}$ has just $\frac{1}{2}(n-1) n^{2}$ independent components, so $K_{\mu \nu \alpha}$ or $\pi_{\mu \mid \nu \alpha}$ can be represented by $\mathcal{T}_{\mu \mid \nu \alpha}$. 
Theorem 2 For post-metric connections we have the following relations

$$
\begin{aligned}
\pi_{\mu \mid \nu \alpha} & =\mathcal{T}_{\nu \mid \alpha \mu}+\mathcal{T}_{\alpha \mid \nu \mu} \\
K_{\mu \nu \alpha} & =\mathcal{T}_{\nu \mid \alpha \mu}+\mathcal{T}_{\alpha \mid \nu \mu}+\mathcal{T}_{\mu \mid \nu \alpha} \\
\mathcal{T}_{\mu \mid \nu \alpha} & =\frac{1}{3}\left(\pi_{\alpha \mid \mu \nu}-\pi_{\nu \mid \mu \alpha}\right)+\widetilde{\mathcal{T}}_{\mu \nu \alpha}
\end{aligned}
$$

and consistent condition

$$
\pi_{\mu \mid \nu \alpha}+\pi_{\alpha \mid \mu \nu}+\pi_{\nu \mid \alpha \mu}=0
$$

$\widetilde{\mathcal{T}}=\widetilde{\mathcal{T}}_{\mu \nu \omega} \gamma^{\mu \nu \omega} \in \Lambda^{3}$ is an arbitrary skew-symmetrical tensor.

Proof If we represent $\pi_{\mu \mid \nu \alpha}$ by $\mathcal{T}_{\mu \mid \nu \alpha}$, by (2.39) and symmetry we have solution as $(2.40)$. By (2.40) we get consistent condition (2.43). By (2.40) and (2.38), we get (2.41).

If we represent $\mathcal{T}_{\mu \mid \nu \alpha}$ by $\pi_{\mu \mid \nu \alpha}$, we generally have linear relation

$$
\mathcal{T}_{\mu \mid \nu \alpha}=k\left(\pi_{\nu \mid \mu \alpha}-\pi_{\alpha \mid \mu \nu}\right)+\widetilde{\mathcal{T}}_{\mu \nu \alpha}
$$

in which $k$ is a constant to be determined, $\widetilde{\mathcal{T}}_{\mu \nu \alpha}$ is particular solution as $\pi_{\mu \mid \nu \alpha} \equiv 0$. $\widetilde{\mathcal{T}}_{\mu \nu \alpha}$ satisfies

$$
\widetilde{\mathcal{T}}_{\mu \nu \alpha}=\widetilde{\mathcal{T}}_{\alpha \mu \nu}=\widetilde{\mathcal{T}}_{\nu \alpha \mu}=-\widetilde{\mathcal{T}}_{\mu \alpha \nu}=-\widetilde{\mathcal{T}}_{\nu \mu \alpha}=-\widetilde{\mathcal{T}}_{\alpha \nu \mu}
$$

So this part of torsion is a skew-symmetrical tensor $\widetilde{\mathcal{T}}=\widetilde{\mathcal{T}}_{\mu \nu \omega} \gamma^{\mu \nu \omega} \in \Lambda^{3}$, which has $C_{n}^{3}=\frac{1}{6}(n-$ $2)(n-1) n$ independent components. Substituting (2.44) into (2.39), we get

$$
(k-1)\left(\pi_{\mu \mid \nu \alpha}+\pi_{\nu \mid \mu \alpha}\right)=2 k \pi_{\alpha \mid \mu \nu}
$$

Calculating the summation of (2.46) for circulation of $\{\mu, \nu, \alpha\}$, we also get consistent condition (2.43). Substituting (2.43) into (2.46) we get $k=\frac{1}{3}$. Again by (2.44), we get solution (2.42). It is easy to check, (2.40) and (2.42) are the inverse representation under condition (2.43). The proof is finished.

Substituting (2.33) into

$$
0=d \mathbf{g}=\delta_{\nu}^{\mu}\left[\left(\mathfrak{d}_{\alpha} \gamma_{\mu}\right) \otimes \gamma^{\nu}+\gamma_{\mu} \otimes \mathfrak{d}_{\alpha} \gamma^{\nu}\right] d x^{\alpha}
$$

we get

$$
\mathfrak{d}_{\alpha} \gamma_{\mu}=\left(\Gamma_{\alpha \mu}^{\nu}+\pi_{\alpha \mu}^{\nu}+\mathcal{T}_{\alpha \mu}^{\nu}\right) \gamma_{\nu}
$$


To understand the meaning of $\pi_{\mu \nu}^{\alpha}$ and $\mathcal{T}_{\mu \nu}^{\alpha}$, we examine the influence on geodesic.

$$
\begin{aligned}
\frac{d \mathbf{v}}{d s} & \equiv \frac{d v^{\alpha}}{d s} \gamma_{\alpha}+v^{\alpha} \mathfrak{d}_{\mu} \gamma_{\alpha} v^{\mu}=\left(\frac{d v^{\alpha}}{d s}+\left(\Gamma_{\mu \nu}^{\alpha}+\pi_{\mu \nu}^{\alpha}+\mathcal{T}_{\mu \nu}^{\alpha}\right) v^{\mu} v^{\nu}\right) \gamma_{\alpha} \\
& =\left(\frac{d}{d s} v^{\alpha}+\Gamma_{\mu \nu}^{\alpha} v^{\mu} v^{\nu}\right) \gamma_{\alpha}+\pi_{\mu \nu}^{\alpha} v^{\mu} v^{\nu} \gamma_{\alpha} .
\end{aligned}
$$

The term $\mathcal{T}_{\mu \nu}^{\alpha} v^{\mu} v^{\nu}=0$ due to $\mathcal{T}_{\mu \nu}^{\alpha}=-\mathcal{T}_{\nu \mu}^{\alpha}$. So the symmetrical part $\pi_{\mu \nu}^{\alpha}$ influences the geodesic, but the antisymmetrical part $\mathcal{T}_{\mu \nu}^{\alpha}$ only influences spin of a particle. This means $\pi_{\mu \nu}^{\alpha} \neq 0$ violates Einstein's equivalent principle. In what follows, we take $\pi_{\mu \nu}^{\alpha}=0$.

By (2.33) and (2.48), we get

Theorem 3 In the case $\pi_{\mu \nu}^{\alpha} \equiv 0$, the absolute differential of vector $\boldsymbol{A}$ is given by

$$
d \boldsymbol{A}=\nabla_{\alpha} A^{\mu} \gamma_{\mu} d x^{\alpha}=\nabla_{\alpha} A_{\mu} \gamma^{\mu} d x^{\alpha}
$$

in which $\nabla_{\alpha}$ denotes the absolute derivatives of vector defined as follows

$$
\begin{gathered}
\nabla_{\alpha} A^{\mu}=A_{; \alpha}^{\mu}+\mathcal{T}_{\alpha \beta}^{\mu} A^{\beta}, \quad A_{; \alpha}^{\mu}=\partial_{\alpha} A^{\mu}+\Gamma_{\alpha \nu}^{\mu} A^{\nu} \\
\nabla_{\alpha} A_{\mu}=A_{\mu ; \alpha}-\mathcal{T}_{\alpha \mu}^{\beta} A_{\beta}, \quad A_{\mu ; \alpha}=\partial_{\alpha} A_{\mu}-\Gamma_{\alpha \mu}^{\nu} A_{\nu}
\end{gathered}
$$

where $A_{; \alpha}^{\mu}$ and $A_{\mu ; \alpha}$ are usual covariant derivatives of vector without torsion. Torsion $\mathcal{T}_{\mu \nu \omega} \in \Lambda^{3}$ is an antisymmetrical tensor of $C_{n}^{3}$ independent components.

Similarly, we can calculate the absolute differential for any tensor. The example also shows the advantages to combine variable with basis.

Now we take spinor connection as example to show the power of Clifford algebra. For Dirac equation in curved space-time without torsion, we have[13-15]

$$
\gamma^{\mu} i\left(\partial_{\mu}+\Gamma_{\mu}\right) \phi=m \phi, \quad \Gamma_{\mu}=\frac{1}{4} \gamma_{\nu}\left(\partial_{\mu} \gamma^{\nu}+\Gamma_{\mu \alpha}^{\nu} \gamma^{\alpha}\right)
$$

$\Gamma_{\mu}$ is called spinor connection. Representing $\gamma^{\mu} \Gamma_{\mu} \in \Lambda^{1} \cup \Lambda^{3}$ in the form of (2.4), we get

$$
\alpha^{\mu} \hat{p}_{\mu} \phi+s_{\mu} \Omega^{\mu} \phi=m \gamma^{0} \phi
$$

where $\alpha^{\mu}$ is current operator, $\hat{p}_{\mu}$ is momentum operator and $s_{\mu}$ spin operator. They are defined respectively by

$$
\alpha^{\mu}=\operatorname{diag}\left(\varrho^{\mu}, \widetilde{\varrho}^{\mu}\right), \quad \hat{p}_{\mu}=i\left(\partial_{\mu}+\Upsilon_{\mu}\right)-e A_{\mu}, \quad s^{\mu}=\frac{1}{2} \operatorname{diag}\left(\varrho^{\mu},-\widetilde{\varrho}^{\mu}\right),
$$


where $\varrho^{\mu}=h_{a}^{\mu} \sigma^{a}, \widetilde{\varrho}^{\mu}=h^{\mu}{ }_{a} \widetilde{\sigma}^{a}$ is the Pauli matrices in curved space-time. $\Upsilon_{\mu} \in \Lambda^{1}$ is called Keller connection, and $\Omega_{\mu} \in \Lambda^{3}$ is called Gu-Nester potential, which is a pseudo vector[13, 15, 16]. They are calculated by

$$
\Upsilon_{\mu}=\frac{1}{2} h_{a}^{\nu}\left(\partial_{\mu} l_{\nu}^{a}-\partial_{\nu} l_{\mu}^{a}\right), \quad \Omega^{\alpha}=\frac{1}{4} \epsilon^{d a b c} h_{d}^{\alpha} h_{a}^{\beta} S_{b c}^{\mu \nu} \partial_{\beta} g_{\mu \nu}, \quad S_{a b}^{\mu \nu} \equiv-h_{[a}^{\{\mu} h_{b]}^{\nu\}} .
$$

In the Hamiltonian of a spinor we get a spin-gravity coupling potential $s_{\mu} \Omega^{\mu}$. If the metric of the space-time can be orthogonalized, we have $\Omega_{\mu} \equiv 0$.

If the gravitational field is generated by a rotating ball, the corresponding metric, like the Kerr one, cannot be diagonalized. In this case, the spin-gravity coupling term has non-zero coupling effect. In asymptotically flat space-time we have the line element in quasi-spherical coordinate system[17]

$$
\begin{gathered}
d \mathbf{x}=\gamma_{0} \sqrt{U}(d t+W d \varphi)+\sqrt{V}\left(\gamma_{1} d r+\gamma_{2} r d \theta\right)+\gamma_{3} \sqrt{U^{-1}} r \sin \theta d \varphi \\
d \mathbf{x}^{2}=U(d t+W d \varphi)^{2}-V\left(d r^{2}+r^{2} d \theta^{2}\right)-U^{-1} r^{2} \sin ^{2} \theta d \varphi^{2}
\end{gathered}
$$

in which $(U, V, W)$ is just functions of $(r, \theta)$. As $r \rightarrow \infty$ we have

$$
U \rightarrow 1-\frac{2 m}{r}, \quad W \rightarrow \frac{4 L}{r} \sin ^{2} \theta, \quad V \rightarrow 1+\frac{2 m}{r},
$$

where $(m, L)$ are mass and angular momentum of the star respectively. For common stars and planets we always have $r \gg m \gg L$. For example, we have $m \doteq 3 \mathrm{~km}$ for the sun. The nonzero tetrad coefficients of metric (2.57) are given by

$$
\left\{\begin{array}{l}
l_{t}^{0}=\sqrt{U}, l_{r}{ }^{1}=\sqrt{V}, l_{\theta}^{2}=r \sqrt{V}, l_{\varphi}^{3}=\frac{r \sin \theta}{\sqrt{U}}, l_{\varphi}^{0}=\sqrt{U} W, \\
h_{0}^{t}=\frac{1}{\sqrt{U}}, h_{1}^{r}=\frac{1}{\sqrt{V}}, h_{2}^{\theta}=\frac{1}{r \sqrt{V}}, h_{3}^{\varphi}=\frac{\sqrt{U}}{r \sin \theta}, h^{t}{ }_{3}=\frac{-\sqrt{U} W}{r \sin \theta} .
\end{array}\right.
$$

Substituting it into (2.56) we get

$$
\begin{aligned}
\Omega^{\alpha} & =h_{0}^{t} h_{1}^{r} h_{2}^{\theta} h_{3}^{\varphi}\left(0, \partial_{\theta} g_{t \varphi},-\partial_{r} g_{t \varphi}, 0\right) \\
& =\left(V r^{2} \sin \theta\right)^{-1}\left(0, \partial_{\theta}(U W),-\partial_{r}(U W), 0\right) \\
& \rightarrow \frac{4 L}{r^{4}}(0,2 r \cos \theta, \sin \theta, 0) .
\end{aligned}
$$

By (2.61) we find that, the intensity of $\Omega^{\alpha}$ is proportional to the angular momentum of the star. By (2.61) we have

$$
\frac{d x^{\mu}}{d s}=\Omega^{\mu} \Rightarrow \frac{d r}{d \theta}=\frac{2 r \cos \theta}{\sin \theta} \Leftrightarrow r=R \sin ^{2} \theta
$$

(2.62) shows that, the flux lines of $\Omega^{\alpha}$ is just the magnetic lines of a magnetic dipole. According to the above results, we know that the spin-gravity coupling potential of charged particles will certainly induce a macroscopic dipolar magnetic field for a star, and it should be approximately in accordance with the Schuster-Wilson-Blackett relation[18-20]. 


\section{REPRESENTATION OF CLIFFORD ALGEBRA}

The matrix representation of Clifford algebra is an old problem with a long history. As early as in 1908, Cartan got the following periodicity of $8[10,11]$.

Theorem 4 For real universal Clifford algebra $C \ell_{p, q}$, we have the following isomorphism

$$
C \ell_{p, q} \cong\left\{\begin{array}{lll}
\operatorname{Mat}\left(2^{\frac{n}{2}}, \mathbb{R}\right), & \text { if } & \bmod (p-q, 8)=0,2 \\
\operatorname{Mat}\left(2^{\frac{n-1}{2}}, \mathbb{R}\right) \oplus \operatorname{Mat}\left(2^{\frac{n-1}{2}}, \mathbb{R}\right), & \text { if } & \bmod (p-q, 8)=1 \\
\operatorname{Mat}\left(2^{\frac{n-1}{2}}, \mathbb{C}\right), & \text { if } & \bmod (p-q, 8)=3,7 \\
\operatorname{Mat}\left(2^{\frac{n-2}{2}}, \mathbb{H}\right), & \text { if } & \bmod (p-q, 8)=4,6 \\
\operatorname{Mat}\left(2^{\frac{n-3}{2}}, \mathbb{H}\right) \oplus \operatorname{Mat}\left(2^{\frac{n-3}{2}}, \mathbb{H}\right), & \text { if } & \bmod (p-q, 8)=5
\end{array}\right.
$$

For $C \ell_{0,2}$, we have $C=t I+x \gamma_{1}+y \gamma_{2}+z \gamma_{12}$ with

$$
\gamma_{1}^{2}=\gamma_{2}^{2}=\gamma_{12}^{2}=-1, \gamma_{1} \gamma_{2}=-\gamma_{2} \gamma_{1}=\gamma_{12}, \gamma_{2} \gamma_{12}=-\gamma_{12} \gamma_{2}=\gamma_{1}, \gamma_{12} \gamma_{1}=-\gamma_{1} \gamma_{12}=\gamma_{2}
$$

By (3.2) we find $C$ is equivalent to a quaternion, that is we have isomorphic relation $C \ell_{0,2} \cong \mathbb{H}$.

Similarly, for $C \ell_{2,0}$ we have $C=t I+x \gamma_{1}+y \gamma_{2}+z \gamma_{12}$ with

$$
\gamma_{1}^{2}=\gamma_{2}^{2}=\gamma_{12}^{2}=1, \gamma_{1} \gamma_{2}=-\gamma_{2} \gamma_{1}=\gamma_{12}, \gamma_{2} \gamma_{12}=-\gamma_{12} \gamma_{2}=-\gamma_{1}, \gamma_{12} \gamma_{1}=-\gamma_{1} \gamma_{12}=-\gamma_{2}
$$

By (3.3), the basis is equivalent to

$$
\gamma_{1}=\left(\begin{array}{cc}
0 & 1 \\
1 & 0
\end{array}\right), \quad \gamma_{2}=\left(\begin{array}{cc}
1 & 0 \\
0 & -1
\end{array}\right), \quad \gamma_{12}=\left(\begin{array}{cc}
0 & -1 \\
1 & 0
\end{array}\right)
$$

Thus (3.4) means $C \ell_{2,0} \cong \operatorname{Mat}(2, \mathbb{R})$.

In geometry and physics, the matrix representation of generators of Clifford algebra is more important and fundamental than the representation of whole algebra. Define $\gamma^{\mu}$ by

$$
\gamma^{\mu}=\left(\begin{array}{cc}
0 & \widetilde{\vartheta}^{\mu} \\
\vartheta^{\mu} & 0
\end{array}\right) \equiv \Gamma^{\mu}(m), \quad \vartheta_{\mu}=\operatorname{diag}(\overbrace{\sigma_{\mu}, \sigma_{\mu}, \cdots, \sigma_{\mu}}^{m}), \quad \widetilde{\vartheta}_{\mu}=\operatorname{diag}(\overbrace{\widetilde{\sigma}_{\mu}, \widetilde{\sigma}_{\mu}, \cdots, \widetilde{\sigma}_{\mu}}^{m}) .
$$

which forms the generator or grade- 1 basis of Clifford algebra $C \ell_{1,3}$. To denote $\gamma^{\mu}$ by $\Gamma^{\mu}(m)$ is for the convenience of representation of high dimensional Clifford algebra. For any matrices $C^{\mu}$ satisfying $C \ell_{1,3}$ Clifford algebra, we have[12, 21]

Theorem 5 Assuming the matrices $C^{\mu}$ satisfy anti-commutative relation of $C \ell_{1,3}$

$$
C^{\mu} C^{\nu}+C^{\nu} C^{\mu}=2 \eta^{\mu \nu}
$$

then there is a natural number $m$ and an invertible matrix $K$, such that $K^{-1} C^{\mu} K=\Gamma^{\mu}(m)$. 
This means in equivalent sense, we have unique representation (3.5) for generator of $C \ell_{1,3}$. In [12], we derived complex representation of generators of $C \ell_{p, q}$ based on Thm.5 and real representations according to the complex representations as follows.

\section{Theorem 6 Let}

$$
\gamma^{5}=i \operatorname{diag}(E,-E), \quad E \equiv \operatorname{diag}\left(I_{2 k},-I_{2 l}\right), \quad k+l=n
$$

Other $\gamma^{\mu},(\mu \leq 3)$ are given by (3.5). Then the generators of Clifford algebra $C \ell_{1,4}$ are equivalent to $\forall \gamma^{\mu},(\mu=0,1,2,3,5)$.

In order to express the general representation of generators, we introduce some simple notations. $I_{m}$ stands for $m \times m$ unit matrix. For any matrix $A=\left(A_{a b}\right)$, denote block matrix

$$
A \otimes I_{m}=\left(A_{a b} I_{m}\right), \quad[A, B, C, \cdots]=\operatorname{diag}(A, B, C, \cdots)
$$

In which the direct product of matrix is Kronecker product. Obviously, we have $I_{2} \otimes I_{2}=I_{4}$, $I_{2} \otimes I_{2} \otimes I_{2}=I_{8}$ and so on. In what follows, we use $\Gamma^{\mu}(m)$ defined in (3.5). For $\mu \in\{0,1,2,3\}$, $\Gamma^{\mu}(m)$ is $4 m \times 4 m$ matrix, which constitute the generator of $C \ell_{1,3}$. Similarly to the above proofs, we can check the following theorem by method of induction.

Theorem $71^{\circ}$ In equivalent sense, for $C \ell_{4 m}$, the matrix representation of generators is uniquely given by

$$
\begin{gathered}
\left\{\Gamma^{\mu}(n),\left[\Gamma^{\mu}\left(\frac{n}{2^{2}}\right),-\Gamma^{\mu}\left(\frac{n}{2^{2}}\right)\right] \otimes I_{2},\right. \\
{\left[\left[\Gamma^{\mu}\left(\frac{n}{2^{4}}\right),-\Gamma^{\mu}\left(\frac{n}{2^{4}}\right)\right],-\left[\Gamma^{\mu}\left(\frac{n}{2^{4}}\right),-\Gamma^{\mu}\left(\frac{n}{2^{4}}\right)\right]\right] \otimes I_{2^{2}},} \\
\left.\left[\Gamma^{\mu}\left(\frac{n}{2^{6}}\right),-\Gamma^{\mu}\left(\frac{n}{2^{6}}\right),-\Gamma^{\mu}\left(\frac{n}{2^{6}}\right), \Gamma^{\mu}\left(\frac{n}{2^{6}}\right),-\Gamma^{\mu}\left(\frac{n}{2^{6}}\right), \Gamma^{\mu}\left(\frac{n}{2^{6}}\right), \Gamma^{\mu}\left(\frac{n}{2^{6}}\right),-\Gamma^{\mu}\left(\frac{n}{2^{6}}\right)\right] \otimes I_{2^{3}}, \cdots\right\} .
\end{gathered}
$$

In which $n=2^{m-1} N, N$ is any given positive integer. All matrices are $2^{m+1} N \times 2^{m+1} N$ type.

$2^{\circ}$ For $C \ell_{4 m+1}$, besides (3.9) we have another real generator

$$
\gamma^{4 m+1}=[E,-E,-E, E,-E, E, E,-E \cdots], \quad E=\left[I_{2 k},-I_{2 l}\right]
$$

If and only if $k=l$, this representation can be uniquely expanded as generators of $C \ell_{4 m+4}$.

$3^{\circ}$ For any $C \ell_{p, q},\{p, q \mid p+q \leq 4 m, \bmod (p+q, 4) \neq 1\}$, the combination of $p+q$ linear independent generators $\left\{\gamma^{\mu}, i \gamma^{\nu}\right\}$ taking from (3.9) constitutes the complete set of generators. In the case $\{p, q \mid p+q \leq 4 m, \bmod (p+q, 4)=1\}$, besides the combination of $\left\{\gamma^{\mu}, i \gamma^{\nu}\right\}$, we have another normal representation of generator taking the form (3.10) with $k \neq l$. 
$4^{\circ}$ For $C \ell_{m},(m<4)$, we have another $2 \times 2$ Pauli matrix representation for its generators $\left\{\sigma^{1}, \sigma^{2}, \sigma^{3}\right\}$.

Then we get all complex matrix representations for generators of real $C \ell_{p, q}$ explicitly.

The real representation of $C \ell_{p, q}$ can be easily constructed from the above complex representation. In order to get the real representation, we should classify the generators derived above. Let $\mathbf{G}_{c}(n)$ stand for any one set of all complex generators of $C \ell_{n}$ given in Thm.7, and set the coefficients before all $\sigma^{\mu}$ and $\tilde{\sigma}^{\mu}$ as 1 or $i$. Denote $\mathbf{G}_{c+}$ stands for the set of complex generators of $C \ell_{n, 0}$ and $\mathbf{G}_{c-}$ for the set of complex generators of $C \ell_{0, n}$. Then we have

$$
\mathbf{G}_{c}=\mathbf{G}_{c+} \cup \mathbf{G}_{c-}, \quad \mathbf{G}_{c-} \cong i \mathbf{G}_{c+}
$$

By the construction of generators, we have only two kinds of $\gamma^{\mu}$ matrices. One is the matrix with real nonzero elements, and the other is that with imaginary nonzero elements. This is because all nonzero elements of $\sigma^{2}$ are imaginary but all other $\sigma^{\mu}(\forall \mu \neq 2)$ are real. Again assume

$$
\mathbf{G}_{c+}=\mathbf{G}_{r} \cup \mathbf{G}_{i}, \quad \mathbf{G}_{r}=\left\{\gamma_{r}^{\mu} \mid \gamma_{r}^{\mu} \text { is real }\right\}, \quad \mathbf{G}_{i}=\left\{\gamma_{i}^{\mu} \mid \gamma_{i}^{\mu} \text { is imaginary }\right\}
$$

Denote $J_{2}=i \sigma^{2}$, we have $J_{2}^{2}=-I_{2} . J_{2}$ becomes the real matrix representation for imaginary unit $i$. Using the direct products of complex generators with $\left(I_{2}, J_{2}\right)$, we can easily construct the real representation of all generators for $C \ell_{p, q}$ from $\mathbf{G}_{c+}$ as follows.

Theorem $81^{\circ}$ For $C \ell_{n, 0}$, we have real matrix representation of generators as

$$
\boldsymbol{G}_{r+}=\left\{\gamma^{\mu} \otimes I_{2}\left(\text { if } \gamma^{\mu} \in \boldsymbol{G}_{r}\right) ; \quad i \gamma^{\nu} \otimes J_{2}\left(\text { if } \gamma^{\nu} \in \boldsymbol{G}_{i}\right)\right\}
$$

$2^{\circ}$ For $C \ell_{0, n}$, we have real matrix representation of generators as

$$
\mathbf{G}_{r-}=\left\{\gamma^{\mu} \otimes J_{2} \mid \gamma^{\mu} \in \boldsymbol{G}_{r+}\right\}
$$

$3^{\circ}$ For $C \ell_{p, q}$, we have real matrix representation of generators as

$$
\boldsymbol{G}_{r}=\left\{\begin{array}{c|l}
\Gamma_{+}^{\mu_{a}}, \Gamma_{-}^{\nu_{b}} & \begin{array}{l}
\Gamma_{+}^{\mu_{a}}=\gamma^{\mu_{a}} \in \boldsymbol{G}_{r+}, \quad(a=1,2, \cdots, p) \\
\Gamma_{-}^{\nu_{b}}=\gamma^{\nu_{b}} \in \boldsymbol{G}_{r-}, \quad(b=1,2, \cdots, q)
\end{array}
\end{array}\right\} .
$$

Obviously we have $C_{n}^{p} C_{n}^{q}=\left(C_{n}^{p}\right)^{2}$ choices for the real generators of $C \ell_{p, q}$ from each complex representation.

Proof. By calculating rules of block matrix, it is easy to check the following relations

$$
\begin{aligned}
& \left(\gamma^{\mu} \otimes I_{2}\right)\left(\gamma^{\nu} \otimes J_{2}\right)+\left(\gamma^{\nu} \otimes J_{2}\right)\left(\gamma^{\mu} \otimes I_{2}\right)=\left(\gamma^{\mu} \gamma^{\nu}+\gamma^{\nu} \gamma^{\mu}\right) \otimes J_{2} \\
& \left(\gamma^{\mu} \otimes J_{2}\right)\left(\gamma^{\nu} \otimes J_{2}\right)+\left(\gamma^{\nu} \otimes J_{2}\right)\left(\gamma^{\mu} \otimes J_{2}\right)=-\left(\gamma^{\mu} \gamma^{\nu}+\gamma^{\nu} \gamma^{\mu}\right) \otimes I_{2}
\end{aligned}
$$


By these relations, Thm.8 becomes a direct result of Thm.7.

For example, we have $4 \times 4$ real matrix representation for generators of $C \ell_{0,3}$ as

$$
\begin{gathered}
i\left\{\sigma^{1}, \sigma^{2}, \sigma^{3}\right\} \cong\left\{\sigma^{1} \otimes J_{2}, i \sigma^{2} \otimes I_{2}, \sigma^{3} \otimes J_{2}\right\} \equiv\left\{\Sigma^{1}, \Sigma^{2}, \Sigma^{3}\right\}= \\
\left\{\left(\begin{array}{cccc}
0 & 0 & 0 & 1 \\
0 & 0 & -1 & 0 \\
0 & 1 & 0 & 0 \\
-1 & 0 & 0 & 0
\end{array}\right), \quad\left(\begin{array}{cccc}
0 & 0 & 1 & 0 \\
0 & 0 & 0 & 1 \\
-1 & 0 & 0 & 0 \\
0 & -1 & 0 & 0
\end{array}\right), \quad\left(\begin{array}{cccc}
0 & 1 & 0 & 0 \\
-1 & 0 & 0 & 0 \\
0 & 0 & 0 & -1 \\
0 & 0 & 1 & 0
\end{array}\right)\right.
\end{gathered}
$$

It is easy to check

$$
\Sigma^{k} \Sigma^{l}+\Sigma^{l} \Sigma^{k}=-2 \delta^{k l}, \quad \Sigma^{k} \Sigma^{l}-\Sigma^{l} \Sigma^{k}=2 \epsilon^{k l m} \Sigma_{m}
$$

\section{TRANSFORMATION OF CLIFFORD ALGEBRA}

Assume $V$ is the base vector space of $C \ell_{p, q}$, then Clifford algebra has the following global properties[22, 23]:

$$
\begin{aligned}
C \ell_{p, q} & =\bigoplus_{k=0}^{n} \Lambda^{k} V=C \ell_{p, q}^{+} \oplus C \ell_{p, q}^{-} \\
C \ell_{p, q}^{+} & \equiv \bigoplus_{k=\text { even }} \Lambda^{k} V, \quad C \ell_{p, q}^{-} \equiv \bigoplus_{k=\text { odd }} \Lambda^{k} V \\
C \ell_{p, q} & \cong C \ell_{p, q+1}^{+} .
\end{aligned}
$$

$C \ell_{p, q}$ is a $\mathbb{Z}_{2}$-graded superalgebra, and $C \ell_{p, q}^{+}$is a subalgebra of $C \ell_{p, q}$. We have

$$
C \ell^{+} C \ell^{+}=C \ell^{-} C \ell^{-}=C \ell^{+}, \quad C \ell^{+} C \ell^{-}=C \ell^{-} C \ell^{+}=C \ell^{-}
$$

Definition 3 The conjugation of element in $C \ell_{p, q}$ is defined by

$$
\left(\gamma_{k_{1} k_{2} \cdots k_{m}}\right)^{*}=(-1)^{m} \gamma_{k_{m} \cdots k_{2} k_{1}}=(-1)^{\frac{1}{2} m(m+1)} \gamma_{k_{1} k_{2} \cdots k_{m}}, \quad(0 \leq m \leq n)
$$

The main involution of element is defined by

$$
\alpha\left(\gamma_{k_{1} k_{2} \cdots k_{m}}\right)=(-1)^{m} \gamma_{k_{1} k_{2} \cdots k_{m}},(0 \leq m \leq n)
$$

The norm and inverse of element are defined by

$$
\mathcal{N}(\boldsymbol{X}) \equiv \boldsymbol{X} \boldsymbol{X}^{*}, \quad \boldsymbol{X}^{-1}=\boldsymbol{X}^{*} / \mathcal{N}(\boldsymbol{X}) \text { if } \mathcal{N}(\boldsymbol{X}) \neq 0
$$


By the definition, it is easy to check

$$
\begin{gathered}
\gamma_{k}^{*}=-\gamma_{k}, \quad \gamma_{a b}^{*}=-\gamma_{a b}, \quad \gamma_{a b c}^{*}=\gamma_{a b c}, \quad \cdots \\
\alpha\left(\mathbf{x}^{*}\right)=\alpha(\mathbf{x})^{*}, \quad \alpha\left(\gamma_{k}\right)=-\gamma_{k}, \quad \alpha\left(\gamma_{a b}\right)=\gamma_{a b}, \quad \cdots \\
\mathbf{g}^{-1}=\mathbf{g}^{*}, \quad\left\{\mathbf{g}=\mathbf{g}_{1} \mathbf{g}_{2} \cdots \mathbf{g}_{m} \mid \forall \mathbf{g}_{k} \in \Lambda^{1}, \mathcal{N}\left(\mathbf{g}_{k}\right)=1\right\} .
\end{gathered}
$$

Definition 4 The Pin group and Spin group of $C \ell_{p, q}$ are defined by

$$
\begin{aligned}
\operatorname{Pin}_{p, q} & =\left\{\mathbf{g} \in C \ell_{p, q} \mid \mathcal{N}(\mathbf{g})= \pm 1, \alpha(\mathbf{g}) \mathbf{x g}^{*} \in V \forall \mathbf{x} \in V\right\} \\
\operatorname{Spin}_{p, q} & =\left\{\mathbf{g} \in C \ell_{p, q}^{+} \mid \mathcal{N}(\mathbf{g})= \pm 1, \mathbf{g x g}^{*} \in V \forall \mathbf{x} \in V\right\}=P \operatorname{Pin} \cap C \ell^{+} .
\end{aligned}
$$

The transformation $\mathbf{x} \mapsto \alpha(\mathbf{g}) \mathbf{x g}^{*}$ is called sandwich operator. Pin or Spin group consists of two connected components with $\mathcal{N}(\mathbf{g})=1$ or $\mathcal{N}(\mathbf{g})=-1$,

$$
\begin{aligned}
& \operatorname{Spin}_{p, q}^{+}=\left\{\mathbf{g} \in C \ell_{p, q}^{+} \mid \mathcal{N}(\mathbf{g})=+1, \mathbf{g x g}^{*} \in V \forall \mathbf{x} \in V\right\} \\
& \operatorname{Spin}_{p, q}^{-}=\left\{\mathbf{g} \in C \ell_{p, q}^{+} \mid \mathcal{N}(\mathbf{g})=-1, \mathbf{g x g}^{*} \in V \forall \mathbf{x} \in V\right\}
\end{aligned}
$$

For $\forall \mathbf{g} \in \operatorname{Pin}_{p, q}, \mathbf{x} \in V$, the sandwich operator is a linear transformation for vector in $V$,

$$
\mathbf{x}^{\prime}=\alpha(\mathbf{g}) \mathbf{x g}^{*} \Rightarrow X^{\prime}=K X, X=\left(x^{1}, x^{2} \cdots x^{n}\right)^{T}
$$

In all transformation of vector, the reflection and rotation transformations are important in geometry. Here we discuss the transformation in detail. Let $\mathbf{m} \in \Lambda^{1}$ is a unit vector in $V$, the reflection transformation of vector $\mathbf{X} \in \Lambda^{1}$ with respect to $n-1$ dimensional mirror perpendicular to $\mathbf{m}$ is defined by[24]

$$
\mathbf{X}^{\prime}=-\alpha(\mathbf{m}) \mathbf{X} \mathbf{m}^{*}=-\mathbf{m X} \mathbf{m}
$$

Let $\mathbf{m}=\gamma_{a} m^{a}, \mathbf{X}=\gamma_{a} X^{a}$, substituting it into (4.16) and using (2.12), we have

$$
\begin{aligned}
\mathbf{X}^{\prime} & =-\left(\mathbf{m} \odot \mathbf{X}+m^{a} \mathbf{X}^{b} \gamma_{a b}\right) \mathbf{m}=-(\mathbf{m} \odot \mathbf{X}) \mathbf{m}-m^{a} X^{b} m^{c}\left(\gamma_{a b} \gamma_{c}\right) \\
& =-(\mathbf{m} \odot \mathbf{X}) \mathbf{m}-m^{a} X^{b} m^{c}\left(g_{b c} \gamma_{a}-g_{a c} \gamma_{b}+\gamma_{a b c}\right) \\
& =-2(\mathbf{m} \odot \mathbf{X}) \mathbf{m}+\mathbf{X}=\mathbf{X}_{\perp}-\mathbf{X}_{\|} .
\end{aligned}
$$

(4.17) clearly shows the geometrical meaning of reflection. By (4.16) we learn reflection transformation belongs to $\operatorname{Pin}_{p, q}$ group.

The rotation transformation $\mathbf{R} \in \operatorname{Spin}_{p, q}$,

$$
\mathbf{X}^{\prime}=\mathbf{R X R}^{-1}
$$


The group elements of elementary transformation in $\Lambda^{2}$ are given by[25]

$$
\begin{aligned}
\left(\cosh \frac{v_{a b}}{2}+\gamma_{a b} \sinh \frac{v_{a b}}{2}\right)^{-1} & =\left(\cosh \frac{v_{a b}}{2}-\gamma_{a b} \sinh \frac{v_{a b}}{2}\right), v_{a b} \in \mathbb{R}, \\
\left(\cos \frac{\theta_{a b}}{2}+\gamma_{a b} \sin \frac{\theta_{a b}}{2}\right)^{-1} & =\left(\cos \frac{\theta_{a b}}{2}-\gamma_{a b} \sin \frac{\theta_{a b}}{2}\right), \theta_{a b} \in[-\pi, \pi) .
\end{aligned}
$$

The total transformation can be expressed as multiplication of elementary transformations

$$
\mathbf{R}=\prod_{\left\{\eta_{a a} \eta_{b b}=-1\right\}}\left(\cosh \frac{v_{a b}}{2}+\gamma_{a b} \sinh \frac{v_{a b}}{2}\right) \prod_{\left\{\eta_{a a} \eta_{b b}=1\right\}}\left(\cos \frac{\theta_{a b}}{2}+\gamma_{a b} \sin \frac{\theta_{a b}}{2}\right) .
$$

(4.21) has $\frac{1}{2}(n-1) n$ generating elements like $S O(n)$. In (4.21) we have commutative relation

$$
\begin{gathered}
{\left[\cosh \frac{v_{a b}}{2}+\gamma_{a b} \sinh \frac{v_{a b}}{2}, \cos \frac{\theta_{c d}}{2}+\gamma_{c d} \sin \frac{\theta_{c d}}{2}\right]=2 \sinh \frac{v_{a b}}{2} \sin \frac{\theta_{c d}}{2} \gamma_{a b} \odot \gamma_{c d}} \\
{\left[\cos \frac{\theta_{a b}}{2}+\gamma_{a b} \sin \frac{\theta_{a b}}{2}, \cos \frac{\theta_{c d}}{2}+\gamma_{c d} \sin \frac{\theta_{c d}}{2}\right]=2 \sin \frac{\theta_{a b}}{2} \sin \frac{\theta_{c d}}{2} \gamma_{a b} \odot \gamma_{c d}}
\end{gathered}
$$

in which

$$
\gamma_{a b} \odot \gamma_{c d}=\eta_{b c} \gamma_{a d}-\eta_{a c} \gamma_{b d}+\eta_{a d} \gamma_{b c}-\eta_{b d} \gamma_{a c} \in \Lambda^{2}
$$

If $a \neq b \neq c \neq d$, the right hand terms vanish, and then two elementary transformations commute with each other.

$\mathbf{R}$ forms a Lie Group of $\frac{1}{2}(n-1) n$ paraments. In the case $C \ell_{n, 0}$ or $C \ell_{0, n}, \mathbf{R}$ is compact group isomorphic to $S O(n)$. Otherwise $\mathbf{R}$ is noncompact one similar to Lorentz transformation. The infinitesimal generators of the corresponding Lie group is $\gamma_{a b}$, and the Lie algebra is given by

$$
\mathcal{R}=\varepsilon^{a b} \gamma_{a b}, \quad\left[\gamma_{a b}, \gamma_{c d}\right]=2 \gamma_{a b} \odot \gamma_{c d} \in \Lambda^{2}, \quad \forall \varepsilon^{a b} \in \mathbb{R}
$$

Thus, $\Lambda^{2} \mathbb{M}^{p, q}$ is just the Lie algebra of proper Lorentz transformation of the space-time $\mathbb{M}^{p, q}$.

\section{APPLICATION IN CLASSICAL GEOMETRY}

Suppose the basic space of projective geometry is $n$-dimensional Euclidean space $\pi$, and the basis is $\left\{\gamma_{a} \mid a=1,2, \cdots, n\right\}$. The coordinate of point $\mathbf{x}$ is given by $\mathbf{x}=\gamma_{a} x^{a}$. The projective polar is $P$, and its height from the basic space $\pi$ is $h$. The total projective space is $n+1$ dimensional, and an auxiliary basis $\gamma_{n+1}=\gamma_{p}$ is introduced. The coordinate of the polar $P$ is $\mathbf{p}=\gamma_{\mu} p^{\mu}$. In this section we use Greek characters for $n+1$ indices. Assume the unit directional vector of the projective ray is $\mathbf{t}=\gamma_{\mu} t^{\mu}$, the unit normal vector of the image space $\pi^{\prime}$ is $\mathbf{n}=\gamma_{\mu} n^{\mu}$, coordinate in $\pi^{\prime}$ is $\mathbf{y}=\gamma_{\mu} y^{\mu}$ and the intercept of $\pi^{\prime}$ with the $n+1$ coordinate axis is $a$. Then we have

$$
(\mathbf{y}-\mathbf{a}) \odot \mathbf{n}=0, \quad \text { or } \quad \mathbf{y} \odot \mathbf{n}=n_{\mu} y^{\mu}=a n_{p}
$$


The equation of projective ray is given by

$$
\mathbf{s}=\mathbf{p}+\lambda \mathbf{t}
$$

where $\lambda$ is parameter coordinate of the line. In the basic space $\pi$, we have $s^{n+1}=0$ and $\lambda=-h / t^{p}$, so the coordinate of the line in $\pi$ reads

$$
\mathbf{x}=\mathbf{p}-\frac{h}{t^{p}} \mathbf{t}
$$

Let $\mathbf{s}=\mathbf{y}$ and substitute (5.2) into (5.1) we get image equation

$$
\mathbf{y}=\mathbf{p}+\frac{a n_{p}-\mathbf{p} \odot \mathbf{n}}{\mathbf{t} \odot \mathbf{n}} \mathbf{t}, \quad \lambda=\frac{a n_{p}-\mathbf{p} \odot \mathbf{n}}{\mathbf{t} \odot \mathbf{n}} .
$$

In the above equation $\mathbf{t} \odot \mathbf{n} \neq 0$, which means $\mathbf{t}$ cannot be perpendicular to $\mathbf{n}$, otherwise the projection cannot be realized. Eliminating coordinate $\mathbf{t}$ in (5.3) and (5.4), we find the projective transformation $\mathbf{y} \leftrightarrow \mathbf{x}$ is nonlinear. In (5.4), only the parameters $(a, \mathbf{n})$ are related to image space $\pi^{\prime}$, so all geometric variables independent of two parameters $(a, \mathbf{n})$ are projective invariants. In what follows we prove the fundamental theorems of projective geometry by Clifford algebra.

Theorem 9 For 4 different points $\left\{\boldsymbol{y}_{1}, \boldsymbol{y}_{2}, \boldsymbol{y}_{3}, \boldsymbol{y}_{4}\right\}$ on a straight line $L$, the following cross ratio is a projective invariant

$$
(12 ; 34) \equiv \frac{\left|\boldsymbol{y}_{1}-\boldsymbol{y}_{3}\right|}{\left|\boldsymbol{y}_{2}-\boldsymbol{y}_{3}\right|} \cdot \frac{\left|\boldsymbol{y}_{2}-\boldsymbol{y}_{4}\right|}{\left|\boldsymbol{y}_{1}-\boldsymbol{y}_{4}\right|}
$$

Proof Substituting (5.4) into (5.5) we get

$$
(12 ; 34)=\frac{\left|\left(\mathbf{t}_{3} \odot \mathbf{n}\right) \mathbf{t}_{1}-\left(\mathbf{t}_{1} \odot \mathbf{n}\right) \mathbf{t}_{3}\right|}{\left|\left(\mathbf{t}_{3} \odot \mathbf{n}\right) \mathbf{t}_{2}-\left(\mathbf{t}_{2} \odot \mathbf{n}\right) \mathbf{t}_{3}\right|} \cdot \frac{\left|\left(\mathbf{t}_{4} \odot \mathbf{n}\right) \mathbf{t}_{2}-\left(\mathbf{t}_{2} \odot \mathbf{n}\right) \mathbf{t}_{4}\right|}{\left|\left(\mathbf{t}_{4} \odot \mathbf{n}\right) \mathbf{t}_{1}-\left(\mathbf{t}_{1} \odot \mathbf{n}\right) \mathbf{t}_{4}\right|} .
$$

By (2.10) and (2.11), we get

$$
\left(\mathbf{t}_{b} \odot \mathbf{n}\right) \mathbf{t}_{a}-\left(\mathbf{t}_{a} \odot \mathbf{n}\right) \mathbf{t}_{b}=\left(\mathbf{t}_{a} \wedge \mathbf{t}_{b}\right) \odot \mathbf{n}= \pm\left|\mathbf{t}_{a} \wedge \mathbf{t}_{b}\right| \mathbf{m} \odot \mathbf{n}
$$

where $\mathbf{m}$ is the unit normal vector of the plane spanned by $\left(\mathbf{t}_{a}, \mathbf{t}_{b}\right)$, which is independent of the image space $\pi^{\prime}$. Substituting it into (5.6), we get

$$
(12 ; 34)=\frac{\left|\mathbf{t}_{3} \wedge \mathbf{t}_{1}\right|}{\left|\mathbf{t}_{3} \wedge \mathbf{t}_{2}\right|} \cdot \frac{\left|\mathbf{t}_{4} \wedge \mathbf{t}_{2}\right|}{\left|\mathbf{t}_{4} \wedge \mathbf{t}_{1}\right|}
$$

(5.8) is independent of $(a, \mathbf{n})$, so it is a projective invariant. Likewise $(13 ; 24)$ and $(14 ; 23)$ are also projective invariants. The proof is finished. 
Now we examine affine transformation. In this case, the polar $P$ at infinity and the directional vector $\mathbf{t}$ of rays becomes constant vector. The equation of rays is given by $\mathbf{y}=\mathbf{x}+\lambda \mathbf{t}$. Substituting it into (5.1) we get the coordinate transformation from basic space $\pi$ to image space $\pi^{\prime}$,

$$
\mathbf{y}=\mathbf{x}+\frac{a n_{p}-\mathbf{n} \odot \mathbf{x}}{\mathbf{t} \odot \mathbf{n}} \mathbf{t}, \quad \lambda=\frac{a n_{p}-\mathbf{n} \odot \mathbf{x}}{\mathbf{t} \odot \mathbf{n}} .
$$

Since $\mathbf{t}$ and $\mathbf{n}$ are constant vectors for all rays, the affine transformation $\mathbf{y} \leftrightarrow \mathbf{x}$ is linear. A variable independent of $(a, \mathbf{n})$ is an affine invariant.

Theorem 10 Assume $\left\{\mathbf{x}_{1}, \mathbf{x}_{2}, \mathbf{x}_{3}\right\}$ are 3 points on a straight line $L$ in basic space $\pi$, and $\left\{\boldsymbol{y}_{1}, \mathbf{y}_{2}, \mathbf{y}_{3}\right\}$ are respectively their projective images on line $L^{\prime}$ in $\pi^{\prime}$. Then the simple ratio

$$
(12,13) \equiv \frac{\left|\boldsymbol{y}_{2}-\boldsymbol{y}_{1}\right|}{\left|\boldsymbol{y}_{3}-\boldsymbol{y}_{1}\right|}
$$

is an affine invariant.

Proof By equation of transformation (5.9) we get

$$
\mathbf{y}_{k}=\mathbf{x}_{k}+\frac{a n_{p}-\mathbf{n} \odot \mathbf{x}_{k}}{\mathbf{t} \odot \mathbf{n}} \mathbf{t} .
$$

In (5.11), only the parameters $(a, \mathbf{n})$ are related to image space $\pi^{\prime}$. Substituting (5.11) into (5.10) we have

$$
(12,13)=\frac{\left|(\mathbf{t} \odot \mathbf{n})\left(\mathbf{x}_{2}-\mathbf{x}_{1}\right)-\mathbf{n} \odot\left(\mathbf{x}_{2}-\mathbf{x}_{1}\right) \mathbf{t}\right|}{\left|(\mathbf{t} \odot \mathbf{n})\left(\mathbf{x}_{3}-\mathbf{x}_{1}\right)-\mathbf{n} \odot\left(\mathbf{x}_{3}-\mathbf{x}_{1}\right) \mathbf{t}\right|}=\frac{\left|\left(\left(\mathbf{x}_{2}-\mathbf{x}_{1}\right) \wedge \mathbf{t}\right) \odot \mathbf{n}\right|}{\left|\left(\left(\mathbf{x}_{3}-\mathbf{x}_{1}\right) \wedge \mathbf{t}\right) \odot \mathbf{n}\right|} .
$$

Denote the unit directional vector of line $L$ by $\mathbf{k}$, then we have

$$
\mathbf{x}_{2}-\mathbf{x}_{1}= \pm\left|\mathbf{x}_{2}-\mathbf{x}_{1}\right| \mathbf{k}, \quad \mathbf{x}_{3}-\mathbf{x}_{1}= \pm\left|\mathbf{x}_{3}-\mathbf{x}_{1}\right| \mathbf{k}
$$

Substituting them into (5.12) we get

$$
(12,13)=\frac{\left|\mathbf{y}_{2}-\mathbf{y}_{1}\right|}{\left|\mathbf{y}_{3}-\mathbf{y}_{1}\right|}=\frac{\left|\mathbf{x}_{2}-\mathbf{x}_{1}\right|}{\left|\mathbf{x}_{3}-\mathbf{x}_{1}\right|}
$$

This proves the simple ratio $(12,13)$ is an affine invariant. Likewise, we can prove $(12,23)$ and $(13,23)$ are also affine invariants. The proof is finished.

The treatment of image information by computer requires concise and general algebraic representation for geometric modeling as well as fast and robust algebraic algorithm for geometric calculation. Conformal geometry algebra was introduced in this context. By establishing unified covariant algebra representation of classical geometry, the efficient calculation of invariant algebra is realized[26-28]. It provides a unified and concise homogeneous algebraic framework for classical 
geometry and algorithms, which can thus be used for complicated symbolic geometric calculations. This technology is currently widely applied in high-tech fields such as computer graphics, vision calculation, geometric design, robots, etc.

The algebraic representation of a geometric object is homogeneous means that, any two algebraic expressions representing this object differ by only one nonzero factor, and any such algebraic expressions with different nonzero multiple represent the same geometric object. The embedding space provided by conformal geometry algebra for $n$ dimensional Euclidean space is $n+2$ dimensional Minkowski space. Since the orthonormal transformation group of the embedding space is exactly double coverage of the conformal transformation group of the Euclidean space, this model is also called the conformal model. The following is a brief introduction to the basic concepts and representation for geometric objects of conformal geometric algebra. The materials mainly come from literature [26].

In conformal geometry algebra, an additional Minkowski plane $\mathbb{M}^{1,1}$ is attached to $n$ dimensional Euclidean space $\mathbb{R}^{n}, \mathbb{M}^{1,1}$ has an orthonormal basis $\left\{e_{+}, e_{-}\right\}$, which has properties:

$$
e_{+}^{2}=1, \quad e_{-}^{2}=-1, \quad e_{+} \odot e_{-}=0
$$

In practical application, $\left\{e_{+}, e_{-}\right\}$is replaced by null basis $\left\{e_{0}, e\right\}$

$$
e_{0}=\frac{1}{2}\left(e_{-}-e_{+}\right), \quad e=e_{-}+e_{+} .
$$

They satisfy

$$
e_{0}^{2}=e^{2}=0, \quad e \odot e_{0}=-1
$$

A unit pseudo-scalar $E$ for $\mathbb{M}^{1,1}$ is defined by

$$
E=e \wedge e_{0}=e_{+} \wedge e_{-}=e_{+} e_{-}
$$

In conformal geometric algebra, we work with $\mathbb{M}^{n+1,1}=\mathbb{R}^{n} \oplus \mathbb{M}^{1,1}$.

Define the horosphere of $\mathbb{R}^{n}$ by

$$
\mathcal{N}_{e}^{n}=\left\{x \in \mathbb{M}^{n+1,1} \mid x^{2}=0, x \odot e=-1\right\}
$$

$\mathcal{N}_{e}^{n}$ is a homogeneous model of $\mathbb{R}^{n}$. The powerful applications of conformal geometry come from this model. By calculation, for $\forall \mathbf{x} \in \mathbb{R}^{n}$ we have

$$
x=\mathbf{x}+\frac{1}{2} \mathbf{x}^{2} e+e_{0}
$$


which is a bijective mapping $\mathbf{x} \in \mathbb{R}^{n} \leftrightarrow x \in \mathcal{N}_{e}^{n}$, we have $\mathcal{N}_{e}^{n} \cong \mathbb{R}^{n}$. $x$ is referred to as the homogeneous point of $\mathbf{x}$. Clearly $0 \in \mathbb{R}^{n} \leftrightarrow e_{0} \in \mathcal{N}_{e}^{n}$ and $\infty \in \mathbb{R}^{n} \leftrightarrow e \in \mathcal{N}_{e}^{n}$ in homogeneous coordinate.

Now we examine how conformal geometric algebra represents geometric objects. For a line passing through points a and $\mathbf{b}$, we have

$$
e \wedge a \wedge b=e \mathbf{a} \wedge \mathbf{b}+(\mathbf{b}-\mathbf{a}) E
$$

Since $\mathbf{a} \wedge \mathbf{b}=\mathbf{a} \wedge(\mathbf{b}-\mathbf{a})$ is the moment for a line through point $\mathbf{a}$ with tangent $\mathbf{a}-\mathbf{b}, e \wedge \mathbf{a} \wedge \mathbf{b}$ characterizes the line completely.

Again by using (5.20) and (5.21), we get

$$
e \wedge a \wedge b \wedge c=e \mathbf{a} \wedge \mathbf{b} \wedge \mathbf{c}+(\mathbf{b}-\mathbf{a}) \wedge(\mathbf{c}-\mathbf{a}) E
$$

We recognize $\mathbf{a} \wedge \mathbf{b} \wedge \mathbf{c}$ as the moment of a plane with tangent $(\mathbf{b}-\mathbf{a}) \wedge(\mathbf{c}-\mathbf{a})$. Thus $e \wedge a \wedge b \wedge c$ represents a plane through points $\{\mathbf{a}, \mathbf{b}, \mathbf{c}\}$, or, more specifically, the triangle $(2$-simplex $)$ with these points as vertices.

For a sphere with radius $\rho$ and center $\mathbf{p} \in \mathbb{R}^{n}$, we have $(\mathbf{x}-\mathbf{p})^{2}=\rho^{2}$. By $(5.20)$, the equation in terms of homogeneous points becomes

$$
x \odot p=-\frac{1}{2} \rho^{2} .
$$

Using $x \odot e=-1$, we get

$$
x \odot s=0, \quad s=p-\frac{1}{2} \rho^{2} e=\mathbf{p}+e_{0}+\frac{1}{2}\left(\mathbf{p}^{2}-\rho^{2}\right) e,
$$

where

$$
s^{2}=\rho^{2}, \quad e \odot s=-1
$$

From these properties the form (5.24) and center $\mathbf{p}$ can be recovered. Therefore, every sphere in $\mathbb{R}^{n}$ is completely characterized by a unique vector $s \in \mathbb{M}^{n+1,1}$. According to (5.25), $s$ lies outside the null cone. Analysis shows that every such vector determines a sphere.

\section{DISCUSSION AND CONCLUSION}

The examples given above are limited in the category of geometry, but we have seen the power of Clifford algebra in solving geometrical problems. In fact, Clifford algebra is more widely used in physics. So why does Clifford algebra work so well? As have been seen from the above examples, the power of Clifford algebra comes from the following features: 
1. In the geometry of flat space, the basic concepts are only length, angle, area and volume, which are already implicitly included in the definition of Clifford algebra. So Clifford algebra actually summarizes these contents of classical geometry and algebraize them all.

2. By introducing the concepts of inner, exterior and direct products of vector, Clifford algebra summarizes the operations of scalars, vectors and tensors and therefore represents all the physical variables in classical physics, as only these variables are included in classical physics.

3. By localizing the basis or frame of space-time, Clifford algebra is naturally suitable for the tangent space in a manifold. If the differential $\partial_{\mu}$ and connection operator $\mathfrak{d}_{\mu} \gamma_{\nu}=$ $\left(\Gamma_{\mu \nu}^{\alpha}+\mathcal{T}_{\mu \nu}^{\alpha}\right) \gamma_{\alpha}$ are introduced, Clifford algebra can be used for the whole manifold, so it also contains Riemann geometry.

4. Together with differential and connection operators, Clifford algebra can express all contents of classical physics, including physical variables, differential equations and algebraic operations. Clifford algebra transforms complicated theories and relations into a unified and standard calculus with no more or less contents, and all representations are neat and elegant.

5. If the above contents seem to be very natural, Clifford algebra still has another unusual advantage, that is, it includes the theory of spinor. So Clifford algebra also contains quantum theory and spinor connection. These things are far beyond the human intuition and have some surprisingly mysterious properties.

6. There are many reasons to make Clifford algebra become a unified and efficient language and tool for mathematics, physics and engineering, such as Clifford algebra generalizes real number, complex number, quaternion and vector algebra; Clifford algebra is isomorphic to matrix algebra; the derivative operator $\gamma^{\mu} \nabla_{\mu}$ contains grad, div, curl, etc. But in the author's view, the most important fact should be taking the physical variable and the basis as one entity, such as $\mathbf{g}=g_{\mu \nu} \gamma^{\mu} \otimes \gamma^{\nu}$ and $\mathcal{T}=\mathcal{T}_{\mu \nu \omega} \gamma^{\mu \nu \omega}$. Just because of this seemingly trivial representation, Clifford algebra calculus has become an arithmetic-like operation which can be well understood by everyone.

"But, if geometric algebra is so good, why is it not more widely used?" As D. Hestenes replied in [29]: "Its time will come!" The published geometric algebra literature is more than sufficient to support instruction with geometric algebra at intermediate and advanced levels in physics, mathematics, engineering and computer science. Though few faculty are conversant with geometric 
algebra now, most could easily learn what they need while teaching. At the introductory level geometric algebra textbooks and teacher training will be necessary before geometric algebra can be widely taught in the schools. There is steady progress in this direction, but funding is needed to accelerate it. Malcolm Gladwell has discussed social conditions for a "tipping point" when the spread of an idea suddenly goes viral. Place your bets now on a Tipping Point for Geometric Algebra!

\section{Acknowledgments}

The discussion on torsion is completed under the inspiration and guidance of Prof. James M. Nester. The content of conformal geometric algebra is added according to the suggestion of Dr. Isiah Zaplana.

[1] H. Grassmann, Die Lineale Ausdehnungslehre, ein neuer Zweig derMathematik [The Theory of Linear Extension, a New Branch of Mathematics], (1844).

[2] W. Hamilton, On Quaternions, or on a New System of Imaginaries in Algebra, Philosophical Magazine (1844).

[3] W. Clifford, Application of Grassmann's Extensive Algebra, Amer. J. Math., 1: 350-358(1878).

[4] D. Hestenes, Oersted Medal Lecture 2002: Reforming the Mathematical Language of Physics. Am. J. Phys. 71, 104-121 (2003)

[5] P. Dirac, The Quantum Theory of the Electron, Proc. Roy. Soc. Lond. A117 (1928) 610-624.

[6] E. Cartan, The Theory of Spinors, The M.T.I. Press, Cambridge, MA 1966.

[7] C. Chevalley, The Algebraic Theory of Spinors and Clifford Algebras, Springer, Berlin 1996.

[8] M. Riesz, Clifford Numbers and Spinors, E.F. Bolinder and P. Lounesto (Eds), Springer, Netherlands 1993.

[9] D. Hestenes, Space Time Algebra. Gordon and Breach, New York (1966)

[10] P. Lounesto, Clifford Algebras and Spinors, Cambridge Univ. Press, Cambridge 2001.

[11] D. S. Shirokov, Clifford algebras and their applications to Lie groups and spinors, Proceedings of the Nineteenth International Conference on Geometry, Integrability and Quantization, Ivailo M. Mladenov and Akira Yoshioka, eds. (Sofia: Avangard Prima, 2018), 11-53, arXiv:1709.06608

[12] Y. Q. Gu, A Note on the Representation of Clifford Algebra, to appear.

[13] Y. Q. Gu, Space-Time Geometry and Some Applications of Clifford Algebra in Physics. Adv. Appl. Clifford Algebras, 2018, 28(4):79.

[14] P. G. Bergmann, Two-Component Spinors in General Relativity, Phys. Rev. 107(2), 624-629 (1957) 
[15] Y. Q. Gu, The Simplification of Spinor Connection and Classical Approximation, arXiv:gr-qc/0610001

[16] J. M. Nester, Special orthonormal frames, J. Math. Phys. 33, 910 (1992).

[17] Y. Q. Gu, The Series Solution to the Metric of Stationary Vacuum with Axisymmetry. Chinese Physics B, 2008, 19(3):90-100.

[18] P. M. S. Blackett, The magnetic field of massive rotating bodies, Nature 159, 658-666 (1947)

[19] A. Dolginov, Electromagnetic Field Created by Rotation of Celestial Bodies. Journal of Modern Physics, 2016, 7(16):2418-2425.

[20] Y. Q. Gu, A New Explanation for the Origin of Magnetic Field of Celestial Bodies. Natural Science(in Chinese), 2019, 7(6):464-470.

[21] Y. Q. Gu, A Canonical Form For Relativistic Dynamic Equation, Adv. Appl. Clifford Algebras, V7(1): 13-24(1997),

[22] D. J. H. Garling, Clifford Algebras: An Introduction, Cambridge Univ. Press, Cambridge 2011.

[23] H. B. Lawson, M. L. Michelsohn, Spin Geometry, Princeton University Press, Princeton, 1989.

[24] C. G. Gunn, Doing Euclidean Plane Geometry Using Projective Geometric Algebra. Adv. Appl. Clifford Algebras, 27:1203-1232 (2017).

[25] Y. Q. Gu, Clifford Algebra, Lorentz Transformation and Unified Field Theory, Adv. Appl. Clifford Algebras, 28(2):37 (2018).

[26] H. B. Li, D. Hestenes, A. Rockwood, Generalized homogeneous coordinates for computational geometry. In G. Sommer, editor, Geometric Computing with Clifford Algebras, pages 27-60. Springer-Verlag, Heidelberg, 2001.

[27] C. Doran, A. Lasenby, Geometric Algebra for Physicists, Ch.10. Cambridge University Press, Cambridge, 2003.

[28] A. Lasenby, J. Lasenby, R. Wareham, A Covariant Approach to Geometry using Geometric Algebra, Technical Report. University of Cambridge Department of Engineering, Cambridge, UK, 2004.

[29] D. Hestenes, The Genesis of Geometric Algebra: A Personal Retrospective. Adv. Appl. Clifford Algebras, 2017, 27(1):351-379. 